\title{
CANCER OF THE BREAST.
}

\author{
By RONALD W. RAVEN, F.R.C.S.
}

(Surgeon to Out-Patients, French Hospital, London; Assistant Surgeon, Royal Cancer Hospital (Free); Assistant Surgeon, Gordon Hospital.)

During recent years important developments have taken place in the treatment of cancer of the breast. This fact is obvious if reference is made to treatises on the subject which were published several years ago, for example "Cancer of the Breast" by Charles Barrett Lockwood written in the year rgI3. He has set out in his own attractive way an experience of a series of operations and their results. The clinical section of the book portrays the clinical acumen of the astute observers of that generation. Lockwood also deals fully with the operative treatment of this disease but it is obvious that with the advances which have been made in methods of irradiation, the scope of therapeutic measures has considerably widened. Evolutionary changes have been taking place in treatment and correlation is being effected between certain clinical types of the disease and the particular form of treatment to be instituted in each group. It can be said that at the present time many types of case considered unsuitable for treatment in the past can be offered therapy with, to say the least, good hope of amelioration.

The object of this communication is to indicate the salient principles of the modern treatment of cancer of the breast and to show the methods of treatment which are indicated for the main clinical types of the disease. The opportunity is also taken to review certain clinical aspects of cancer of the breast with special reference to symptomatology and metastasia.

Attention is also called to the agency by which these important developments have taken place, that is by a team of workers acting together in close consultation and co-operation.

\section{Team-work in Cancer Therapy.}

In no other form of medical practice is team-work of greater importance than in the study and treatment of cancer. The problems associated with the early diagnosis and treatment of the disease are so many and so detailed as to be beyond the scope of any one man to deal with. Whilst these statements apply to cancer therapy in general, it is equally true of, and applies with equal force to, the treatment of cancer of the breast. Each member of the team is important and has his own particular part to play. The registrar is entrusted to keep full and accurate case-records of every patient. Such a case-record must embody the present, past and family histories and personal habits of the patient together with accurate details of investigations carried out, treatment given and the subsequent history of the patient in the follow-up department. It will be from a study of these scientific documents that we shall learn more of the natural history of the disease and its treatment. An expert diagnostic radiologist must be available to investigate manifestations of the disease in other regions of the body. The clinician in the team will require the expert help of the radium therapist and the X-ray therapist to advise on all matters connected with irradiation methods. This idea of team-work in cancer therapy is new but it is believed that the method herein outlined will play a large part in the clinical study of cancer in the future. 


\section{Symptomatology.}

Cancer of the breast is commonest in women between the age of $5^{\circ}$ and 60 years and occurs a little more frequently in unmarried women. The disease occurs with equal frequency in either breast. With regard to the position of the growth in the breast, it appears that the upper and outer quadrant is the commonest site and, after that, the region deep to the nipple. The length of history is variable; some patients present themselves for treatment immediately the lump is discovered in the breast but it is true to say that the disease has been present for at least a year in the majority of patients before they apply for treatment. The commonest symptom is a lump in the breast: in about one-third of patients this lump is painless. Very few patients with cancer of the breast complain of pain in the breast. In general it may be stated that pain as a symptom is of little use in the diagnosis of cancer.

On examination, the lump may be seen in the affected breast as a prominence in the quadrant affected. Careful inspection of the nipple may reveal either deviation towards the growth or definite retraction. These changes are frequently seen in the nipple but discharge and ulceration are less common. Changes may occur in the skin covering the growth-an early change is dimpling and, later, definite puckering and peau d'orange may be seen. In the late stages of the disease the presence of a malignant ulcer is noted. On palpation of the breast a lump is felt with the flat examining hand and the lump is notable for its degree of hardness. This degree of hardness is of great diagnostic importance in cancer of the breast. The edge of the lump is ill-defined and it appears to merge with the breast substance. It is important to notice whether there is any fixation of the lump to the overlying skin or nipple or underlying deep fascia, pectoralis major muscle or the bony components of the chest wall. In certain cases there may also be other isolated nodules of growth in the skin.

A method of examination in cancer of the breast which does not appear to have the prominence it merits is the procedure of trans-illumination. This method is of value especially in doubtful cases with pendulous breasts. The examination room should be darkened and a Cameron light is employed. If a carcinoma is present a shadow is seen in the breast-a normal breast being translucent. If the breast is the site of chronic interstitial mastitis there is no shadow and cysts and abscesses are only partially translucent.

The regional lymph nodes in the axilla and supra-clavicular regions are critically examined. If metastases are present the nodes may be enlarged and hard but it is an error to suppose that no metastases are present in the regional lymph nodes if these are not palpable. The opposite breast and its regional lymph nodes must also be examined.

The general examination of the patient is of importance and special attention must be given to the chest and abdomen. The condition of the skeleton must also be elucidated. The clinical examination is followed by certain radiological investigations. An X-ray examination of the chest should be performed as a routine and if there are any symptoms indicative of involvement of the skeleton an X-ray examination of the suspicious bone must be performed.

\section{The Influence of the Clinical Findings on Treatment.}

The clinical examination of the patient determines the procedure of treatment to be adopted. As a result of this examination it is possible to assign patients to 
certain groups distinguished by the stage of the disease. For purposes of discussion regarding the treatment of cancer of the breast patients may be divided into three groups.

1. Stage I of disease-Carcinoma localised in the breast without attachment to the overlying skin or underlying deep fascia and there are no enlarged regional lymph nodes.

2. Stage II of disease - Carcinoma of the breast with early attachment to skin and/or underlying deep fascia. There are enlarged, hard lymph nodes in the axilla of the same side.

3. Stage III of disease-Carcinoma of the breast with fixation to or ulceration of skin. There is fixation to underlying structures with enlarged, hard, fixed regional lymph nodes. There may also be nodules in the chest wall or metastases in other regions.

For patients with early cancer of the breast (Stage I) it is my practice to recommend the complete operation. The end-results expressed as survival rates at the end of five and ten years may be said to be good. There is a certain proportion of cases in which this method of treatment will fail. Recurrences and metastases occur in the skin, regional lymph nodes and in other structures. This may be due to the fact that the disease was more extensive at the time of the operation than the clinical assessment suggested. If, therefore, the complete operation fails to give permanent relief in a certain proportion of the early cases we must endeavour to find an adjunct to surgical treatment. During recent years there has been a notable advance in methods of irradiation and high voltage X-ray therapy has been employed in the treatment of cases of cancer of the breast. It is my practice in these cases of Stage I cancer of the breast to give a course of high voltage X-ray therapy after the complete operation has been performed. In this group the irradiation is given chiefly to the regional lymph node areas-axillary and supra-clavicular of the same side. If the disease has spread to the regional lymph nodes the prognosis is more problematical for involvement of the regional lymph nodes is the touchstone by which prognosis must be assessed. It is true to say that in a group of such cases about seventy per cent. will be dead at the end of five years with treatment by the complete operation. Metastasia is common in these cases and the problem is how to prevent these metastases and improve the survival rate. In patients with cancer of the breast in Stage II it is my practice to perform the complete operation and to give a complete course of post-operative irradiation with high voltage X-rays delivered to the scar area and to the regional lymph nodes-axillary and supra-clavicular of the same side. It is still a distressing fact that patients are applying for treatment when the disease is far advanced necessitating classification as Stage III on clinical grounds.

This group of cases presents many different and difficult problems. Certain illustrative examples of cases in this group are given in order to clarify the position.

(a) Massive carcinoma of the breast with extensive infiltration of the skin, with or without enlarged regional lymph nodes. This type of case is met with fairly commonly and is unsuitable, in the first instance, for excisional surgical treatment. Since the disease is widespread uniform irradiation over a wide area is essential and a complete course of high voltage $X$-ray therapy to the breast area and regional lymph node areas is advised. The initial results of this form of therapy in these advanced cases are encouraging. The carcinoma in the breast becomes much smaller and localised and the condition of the skin improves. These cases should be reviewed at the end of two months following the cessation of irradiation 
therapy. If there is still a residual mass in the breast and the condition of the skin has improved and there is no evidence of distant metastases it is advisable to perform excision of the breast with the regional axillary lymph nodes. A careful pathological examination is made of the excised breast and lymph nodes, and, if there are metastases in the nodes, a further course of high voltage X-ray therapy is given to the regional lymph node areas.

(b) Localised ulcerating carcinoma of the breast with fixity to deep structures. A patient with this type of cancer of the breast is unsuitable for excisional surgical treatment and some form of irradiation therapy must be instituted. In my experience this variety responds satisfactorily to irradiation from interstitial radium needles. An endeavour is made to ensure irradiation of the whole of the affected breast together with the regional lymph nodes in the axilla and supra-clavicular regions of the same side and also the anterior mediastinum. The radium needles are inserted in a plane deep to the carcinoma in the pectoralis fascia. If the patient is fat, additional radium needles are inserted in a plane nearer the surface of the breast. In this variety of the disease treated in this way the results are often encouraging and healing of the ulcer and prolongation of life are effected. It is advisable later, if there is a residual mobile mass in the breast, to excise the breast with or without the axillary lymph nodes depending on clinical findings.

(c) Cancer of the breast with a single metastasis in bone. If a patient applies for treatment with the disease in this stage, surgical operation is out of the question. It is possible, however, to advise high voltage $\mathrm{X}$-ray therapy for the local breast condition in order to retard its rate of growth. High voltage X-ray therapy may also be given with advantage to the single osseous metastasis. I have seen benefit accrue in such cases and life prolonged from this form of treatment.

(d) Cancer of the breast accompanying lactation. The prognosis with this type of disease is grave whatever treatment is instituted. It is advisable to treat these cases with high voltage X-ray therapy to the breast and regional lymph nodes.

\section{Principles of the Complete Operation for Cancer of the Breast.}

1. Pre-operative care of the patient.-It is essential that care be given to the preparation of the patient for this major surgical procedure. Many of these patients are not good operative risks and the operative mortality is not negligible. It is advisable that the patient should be hospitalized for several days before operation. Ample fluids should be administered and the bowels regulated. The condition of the lungs and kidneys should be estimated and an examination of the blood should be made and anæmia rectified if present.

2. The amount of skin to be removed.-The centre of the growth must be established as the central point of the operation area. An adequate amount of skin must be excised and when making this decision no regard must be paid to the subsequent closure of the wound. The surgeon must ensure that the incision is in healthy tissue wide of the growth. If the resulting wound is large it can be closed by making tension incisions, by flaps, by undercutting, or by skin grafting.

3. The amount of deep fascia to be removed.-The amount of deep fascia removed should be as wide as possible. In general it may be stated that the deep fascia should be removed from the clavicle above, down to a point two inches below the ensiform cartilage, internally two inches beyond the middle line covering the opposite pectoralis major muscle, and, externally, one inch beyond the anterior border of the latissimus dorsi muscle. 
4. Removal of Muscles. - The sternal portion of the pectoralis major muscle and the whole of the pectoralis minor muscle must be removed. It is also necessary to remove every part of the origin of the pectoralis major muscle from the anterior layer of the sheath of the rectus abdominis muscle.

5. Removal of axillary lymphatics, fat and fascia.-A careful dissection of the axilla should be made with the scalpel. The costa-coracoid membrane with the fat and lymphatics are removed and care must be taken to remove the lymphatics and fat from the apex of the axilla. The axillary vein is then systematically dissected from the beginning to the end. The tributaries of the axillary vein are clamped and tied close to it beginning from above downwards, and care must be taken not to include connective tissue in these ligatures. The inner and posterior axillary walls are then cleansed of fat and fascia from above downwards.

6. The mass of tissue finally removed.-This comprises the whole breast with the overlying skin and underlying deep fascia, sternal portion of the pectoralis major and pectoralis minor muscles and the fat, fascia and lymphatics of the axilla. These tissues are removed in one mass.

7. The dressing at the completion of the operation.-The incision is covered with sterilized gauze with plenty of sterilized wool especially in the area where most oozing will occur. In order to obliterate the dead space and to ensure firm fixity of the extensive skin flaps to the chest wall it is advisable to bandage the arm to the chest wall for several days following the operation. Early movements for the arm should be instituted.

\section{The Distribution of Metastases after the Complete Operation.}

Metastases occurring after the complete operation for cancer of the breast may be divided into two groups-

(a) Metastases occurring near the primary disease;

(b) Metastases occurring distant from the primary disease.

(a) Metastases Occurring near the Primary Disease.

As a result of careful follow-up study of large series of cases I have come to regard the following sites as frequently affected by metastases.

(i) The skin of the chest wall in the region of the scar. This region is commonly the site of carcinomatous nodules occurring in the skin or subcutaneous tissues. The occurrence of these nodules may be due to deficient removal of skin with the complete operation. If there is reason to believe that the incisions are not wide of the growth at the time of operation a complete course of post-operative high voltage $\mathrm{X}$-ray therapy should be given to the scar to destroy any malignant cells which may be left behind in these tissues.

(ii) The supra-clavicular lymph nodes of the same side. I have been impressed with the frequency of involvement of these lymph nodes after the complete operation and this is often the first re-manifestation of disease. It has been debated whether or not the supra-clavicular lymph nodes are involved secondarily to the mediastinal lymph nodes. I have studied fourteen patients who had metastases both in the supra-clavicular and mediastinal lymph nodes: in 3 of these cases there was simultaneous involvement; in 7 cases involvement of the mediastinum occurred later at intervals of two months up to two years; in 4 cases the 
mediastinal lymph nodes were affected and several months later there was involvement of the supra-clavicular lymph nodes. Metastases in the supra-clavicular lymph nodes may be associated with massive œdema of the upper extremity of the same side.

(iii) Axillary lymph nodes. The axillary lymph nodes are fairly frequently involved as a re-manifestation of the disease both in the affected and opposite sides.

On account of the frequency of involvement of the supra-clavicular and axillary lymph nodes by disease after the complete operation a complete course of high voltage $\mathrm{X}$-ray therapy is given to the axilla and supra-clavicular areas on the same side.

(iv) Other areas. The opposite breast is not infrequently involved by disease and also the sternum, ribs and costo-chondral junctions.

\section{(b) Metastases Occurring distant from the Primary Disease.}

(i) Bone. Metastases are frequently seen in bone. In a large series of cases I found that the vertebral column is the commonest site for osseous metastases and the lower dorsal and lumbar vertebræ are most often involved. Other bones affected by metastases are the femur, pelvic bones, skull, clavicle and humerus.

(ii) Mediastinum. The lymph nodes in the mediastinum are fairly frequently the site of metastases. It appears that these lymph nodes are more often affected than the lungs and pleura.

(iii) Liver. Metastases are by no means uncommon in the liver after the complete operation for cancer of the breast.

(iv) Other regions. Metastases may occur in the brain, peritoneum, omentum, ovary and retro-orbital tissues.

\section{The Distribution of Metastases after Interstitial Irradiation.}

As a result of studying a large series of patients with cancer of the breast treated by means of interstitial irradiation I have come to the conclusion that there is no greater incidence of metastases in this series than in a series treated by excisional surgery. Metastases frequently occur in the skin of the chest wall and regional lymph nodes. It is important to note that widespread metastases may be present although no sign of cancer can be detected clinically in the breast.

\section{Conclusions.}

I. Attention is called to the importance of team-work in cancer therapy in general and in the therapy of cancer of the breast in particular. 2. The symptomatology of cancer of the breast is reviewed. 3. The salient features of the modern treatment of cancer of the breast are stated. 4. Metastasia in cancer of the breast is discussed with reference to excisional surgery and radium therapy. 